\title{
High-B-Value Diffusion-Weighted Imaging
}

National Cancer Institute

\section{Source}

National Cancer Institute. High-B-Value Diffusion-Weighted Imaging. NCI Thesaurus.

Code C116754.

Diffusion-weighted imaging done under conditions of high diffusion gradients. 